\title{
Evaluation of Battery-free UHF-RFID Sensor Wireless Signals for In-pipe Robotic Applications
}

This paper was downloaded from TechRxiv (https://www.techrxiv.org).

\section{LICENSE}

CC BY 4.0

SUBMISSION DATE / POSTED DATE

03-09-2021 / 09-09-2021

\section{CITATION}

Gunatilake, Amal; Thiyagarajan, Karthick; kodagoda, sarath (2021): Evaluation of Battery-free UHF-RFID Sensor Wireless Signals for In-pipe Robotic Applications. TechRxiv. Preprint. https://doi.org/10.36227/techrxiv.16566111.v1

$\mathrm{DOI}$ 


\section{Evaluation of Battery-free UHF-RFID Sensor Wireless Signals for In-pipe Robotic Applications}

\author{
Amal Gunatilake \\ iPipes Lab, UTS Robotics Institute \\ University of Technology Sydney \\ Sydney, Australia. \\ Amal.Gunatilake@uts.edu.au
}

\author{
Karthick Thiyagarajan \\ iPipes Lab, UTS Robotics Institute \\ University of Technology Sydney \\ Sydney, Australia. \\ Karthick.Thiyagarajan@uts.edu.au
}

\author{
Sarath Kodagoda \\ iPipes Lab, UTS Robotics Institute \\ University of Technology Sydney \\ Sydney, Australia. \\ Sarath.Kodagoda@uts.edu.au
}

\begin{abstract}
A reliable robotic localization method is required for comparing three-dimensional pipe maps obtained via laser scans at various times for accurately monitoring the evolution of internal pipe surface defects. Existing robotic localization methods have limitations when visual features vanish due to changes in the pipe environment or when encoder data becomes highly uncertain due to long-distance robotic traverses. To address this issue, we leverage battery-free ultra-high frequency radio frequency identification (UHF-RFID) sensors for transmitting wireless signals to a two-antenna reader integrated mobile robotic system. Although there are literature on the investigation of UHFRFID behaviors and their applications in indoor environments, analysis of the same for in-pipe scenarios was not well studied. In this paper, we evaluate the UHF-RFID sensor signals inside a field extracted pipeline. Firstly, we examine the UHF-RFID sensor signal patterns through repeated robotic scans. Secondly, we examine how the placement of UHF-RFID reader antennas affects the transmission of UHF-RFID sensor signals, as well as we study the effects of robotic traverse direction and speed on the UHF-RFID wireless signals. Finally, we examine whether identical UHF-RFID sensors generate the same pattern when placed in a pipeline. Overall, the experimental evaluation demonstrates that the use of two-antenna UHF-RFID readers can ameliorate the capabilities of robotic localization in the pipeline.

Index Terms-Battery-free sensors, infrastructure robotics, infrastructure sensing, RFID sensors, robot localization, perception, smart pipes, structural health monitoring, UHF-RFID.
\end{abstract}

\section{INTRODUCTION}

Water utilities worldwide manage millions of kilometres of pipelines that provide residential and industrial customers with water and wastewater services. Corrosion is a significant cause of structural degradation in those pipelines. Water utilities assess corrosion conditions using a variety of sensing technologies [1]-[7] in order to avert catastrophic pipe failures. To extend the useful service life of the pipeline and reduce costs associated with pipe replacement, severely corroded pipes are replaced, while moderately corroded pipes are applied with protective linings [8]. However, if the linings are not applied properly, they could deteriorate in the short-term, and in longterm, surface defects such as folds, wrinkles, bulges, and dimples can occur as a result of the pipe's adverse conditions.

This work was supported by the University of Technology Sydney (UTS) through the Faculty of Engineering and Information Technology (FEIT) Tech Lab Blue Sky Research Grant.

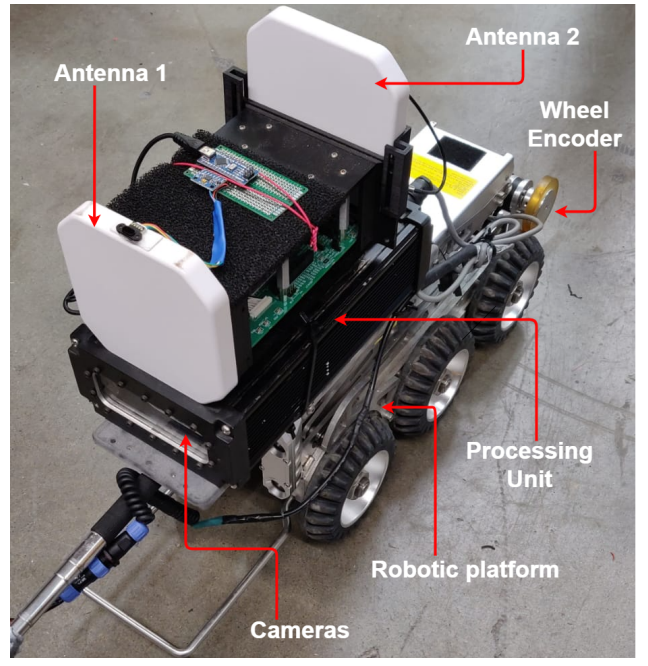

Fig. 1. Mobile robotic system integrated with two UHF-RFID antenna readers.

We have developed a mobile robotic system equipped with a laser profiler for three-dimensional (3D) mapping of water and wastewater pipeline internal surface defects [9], [10]. However, effective robot localization is required to track the evolution of surface defects over time by comparing 3D pipe maps acquired through robotic laser scans at various times. Wheel encoders or tether encoders are the most often utilized localization methods for in-pipe robotic platforms, although they produce significant errors in long-distance traverse owing to wheel skid. Other methods of localization based on visual features have become obsolete due to the changing environmental conditions within pipes as they corrode, are rehabilitated, or are replaced over time.

Localization technologies based on contact-less and wireless sensor signals are ideal for in-pipe robotic applications such that floating robots, mobile robots or drones can be employed. We identified ultra-high frequency radio-frequency identification (UHF-RFID) based localization technology as a promising solution as a result of our literature review because it is less expensive and can be embedded between the host pipe surface and linings. There are various RFID-based robotic localization methods in the literature [11]-[16], but there are limited studies for in-pipe robotic applications. We used a 


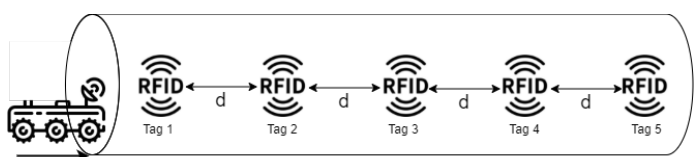

Fig. 2. Illustration of UHF-RFID sensor placement inside the pipe testbed.

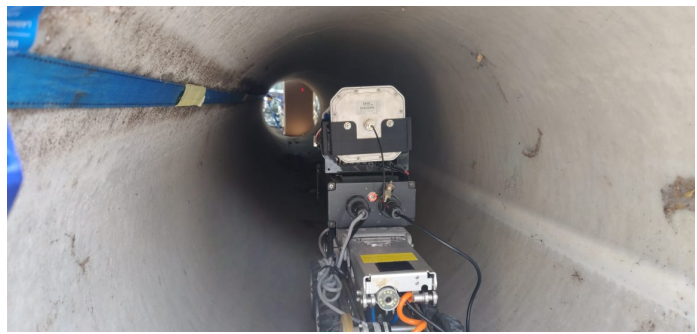

Fig. 3. Robot traversing inside the pipe for collecting UHF-RFID sensor wireless signals.

single UHF-RFID reader antenna model for in-pipe robotic localization in prior work [17], and we were able to obtain the accuracy of up to $15 \mathrm{~cm}$. With the motive of improving robotic localization accuracy, we are focusing on using a mobile robot equipped with two UHF-RFID reader antenna as shown in Fig. 1 for evaluating battery-free UHF-RFID tags as sensors emitting wireless signals inside the field extracted drinking water pipeline. In this paper, firstly, we evaluate the UHFRFID sensor signal patterns with repeated scans. Secondly, we examine the effect of UHF-RFID reader antenna placement, and the direction and speed of mobile robot traverse on UHF-RFID sensor signals. Thirdly, we determine whether identical UHF-RFID sensors produce a similar pattern after being placed inside the pipeline.

\section{System Description And Testbed Overview}

In this work, we leveraged the mini-PIRO robotic tool [9] for integrating UHF-RFID reader antenna and signal processing unit. The UHF-RFID reader antenna was assembled by integrating the Thingmagic M6e Micro-LTE UHF 2 port RFID reader module with the embedded developer kit and two $915 \mathrm{MHz}$ General Purpose Panel RF Antennas in the $902 \mathrm{MHz}$ to $928 \mathrm{MHz}$ range with $5.5 \mathrm{~dB}$ gain. Furthermore, it is actively supported by the open source Python MercuryAPI software community.

We used the Jetson Nano Developer kit board as the central processing unit (CPU), which has a Quad-core ARM $1.43 \mathrm{GHz}$ and 4 GB 64-bit LPDDR4 RAM. The CPU is enclosed in a PVC made casing and mounted on top of a robotic platform as shown in Fig. 1. A rotary encoder with 1024 pulses per revolution was fitted to the robot's wheels to gather odometry data for distance validation.

In the iPipes Lab, a testbed was constructed using a fieldextracted 5-metre long cast iron cement lined pipe to evaluate the battery-free single-chip UHF-RFID sensor wireless signals inside the pipeline, as illustrated in Fig. 2. The UHF-RFID sensors were evenly spaced on the pipe's side wall, and to read the UHF-RFID sensor's wireless signals, the robot traversed within the pipe as illustrated in Fig. 3.

\section{EXPERIMENTS AND RESULTS}

\section{A. UHF-RFID sensor signal evaluation with repeated scans}

This experiment was performed to determine the amount of signal deviation that would occur if we repeated scans of the same configuration with minor changes to the UHFRFID sensor placements. We collected data from five UHFRFID sensors by spacing them one metre apart inside the pipe sample's side wall and operating the robot at a speed of 0.1 metres per second, as shown in Fig. 4. Following that, we randomly positioned the UHF-RFID sensors (within a 10centimetre range from the initial position) and operated the robot a second time to collect data, as illustrated in Fig. 4 round 2 data pattern. The results indicate that the two data patterns are aligned very well. We plotted the difference between the two scans using a histogram to determine the degree of divergence between the two rounds of robotic scans. As shown in Fig. 5, the majority of the data fits within the \pm $2 \mathrm{~dB}$ mean signal variation with 0.89 correlation coefficient. This experiment demonstrates the confidence in the signal's repeatability, which will aid in localization tasks.

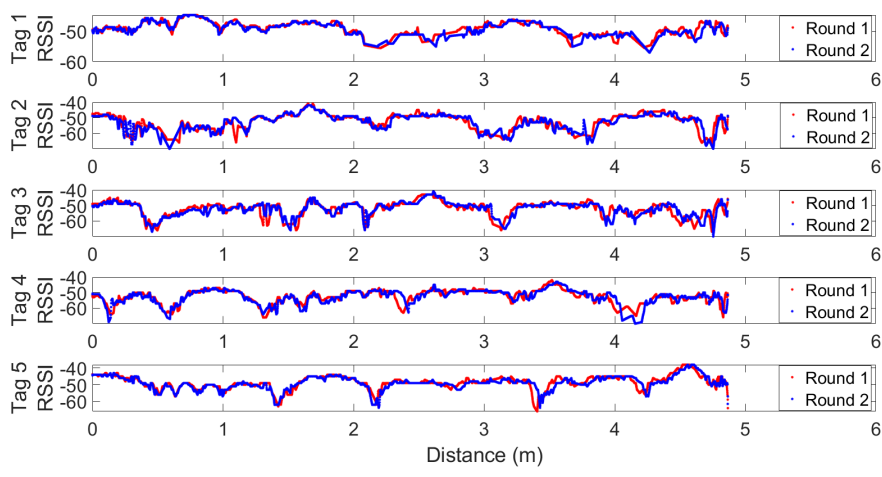

Fig. 4. UHF-RFID sensor wireless signal comparison of two robotic scans.

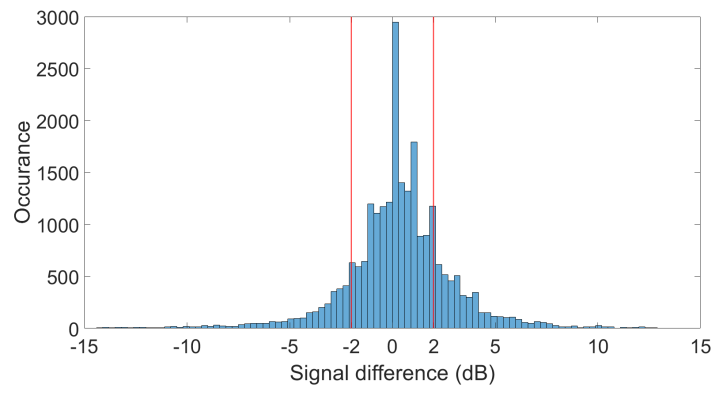

Fig. 5. Histogram of UHF-RFID sensor signal difference for each data point.

We observed in the previous experiment that, having shared the same antenna model, the two UHF-RFID reader antennas produce significantly different signal patterns for the same UHF-RFID sensor. We conducted two identical tests with the two UHF-RFID antennas to determine the signal difference. The next experiment began with one antenna attached to the robot's front and scanning the pipe, and with another test using the same setup but with a second antenna attached to the robot's front and scanning the pipe. The signal difference 
between identical UHF-RFID sensors placed in the pipe is depicted in Fig. 6 with a 0.53 correlation coefficient. Obtaining distinct signal signatures from antennas for the same UHFRFID sensor will significantly aid in localization, as we can multiply the unique data measurements for a specific location as the number of antennas increases.

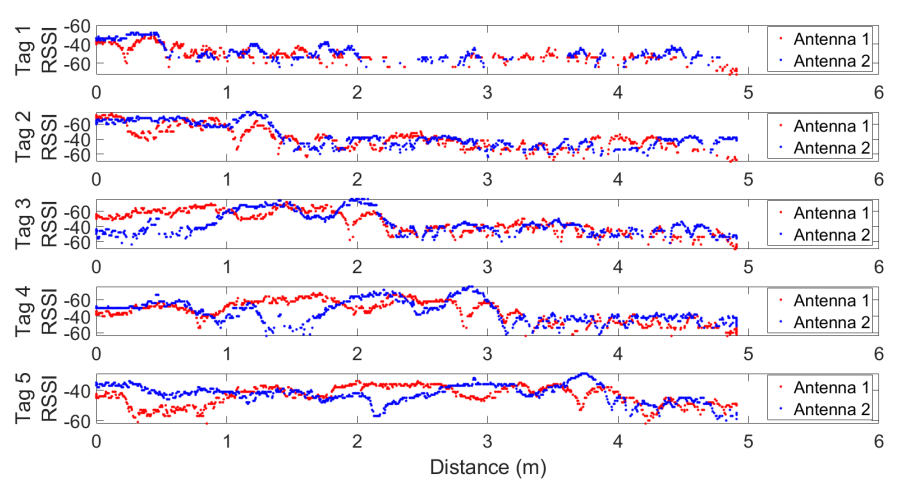

Fig. 6. UHF-RFID sensor signal difference from same model two antennas.

As some research indicates that signal interference between antennas can occur depending on the hardware used to trigger both antennas simultaneously to receive signals [18], we conducted another test to determine if our hardware exhibits any indication of such incidents. We collected data from each antenna separately by running inside the pipe, and then from both antennas simultaneously. By comparing all those sets of data resulting with a 0.92 correlation coefficient, we determined that there is no significant effect on the signal when two antennas operate concurrently in our hardware.

B. Evaluating the effects of UHF-RFID reader antenna direction, robot's direction \& speed on UHF-RFID sensor signals

Firstly, we examined how the placement of the UHF-RFID reader antenna direction affects the UHF-RFID sensor signal pattern. The first scan was completed with the antenna facing forward, and the second scan was completed with the same antenna facing backwards. As shown in Fig. 7, the signal pattern changes as the antenna direction changes, indicating a 0.0468 correlation coefficient. As a result, once the training (initial) data set for robot localization is collected, we should

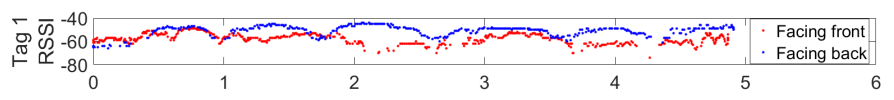

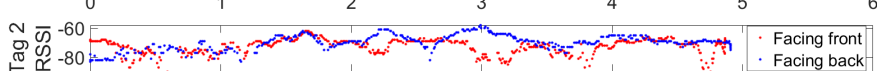

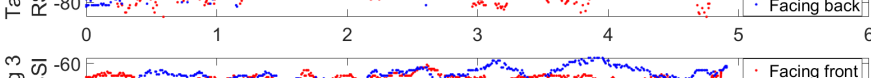

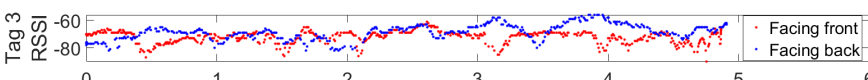

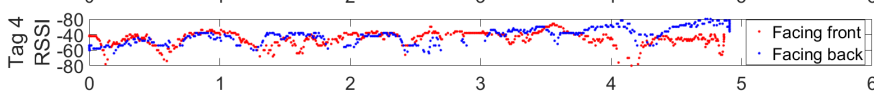

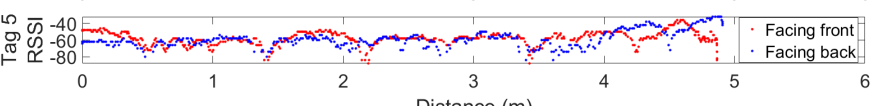
Distance $(m)$

Fig. 7. UHF-RFID sensor signal difference when antenna face opposite direction.

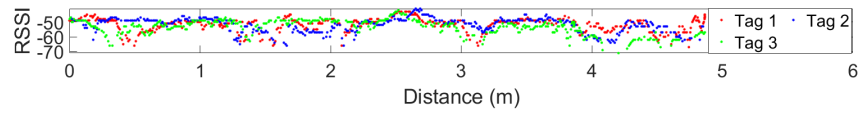

Fig. 8. Wireless signal difference between same family of UHF-RFID sensors

avoid changing the sensor model because this will render the collected data obsolete. Secondly, this experiment was conducted to determine the effect of the robot's movement on the signal. We performed one scan with the robot moving forward and another with the robot moving backwards. We did not adjust the UHF-RFID reader antenna's facing direction in any situation. Following that, we aligned the two scans to visualize the signal difference, which appears as the graph in Fig. 4 indicating a 0.91 correlation coefficient. As a result, it is clear that the direction of travel of the robot has no effect on the UHF-RFID sensor signal pattern. Thirdly, another experiment was conducted to determine how the signal pattern behaved when the robot's speed was varied $(0.1,0.3$, and 0.5 metres per second). The results indicated that driving the robot slower had no effect on the signal pattern, but rather on the data frequency, resulting in higher resolution data.

\section{Evaluating the signals of same type UHF-RFID sensor}

The purpose of this experiment is to determine the signal strength of various UHF-RFID sensors from the same model. We were able to perform independent scans for each sensor by placing them in the middle of the pipe and moving the robot from end to end. The signal difference of three sensors of same type is shown in Fig. 8 with a 0.64 correlation coefficient. Even if the sensors are of the same type, each sensor signal appears to have a slightly different signal pattern when the antenna is not closer (highest peak) to the sensor. The localization task will be aided by having relatively distinctive signal signatures from each UHF-RFID sensor, as it will help to narrow down the search area.

\section{COnClusion And Future Work}

In this paper, we evaluated the battery-free UHF-RFID sensor wireless signals inside a field extracted pipe. Firstly, we repeated scans of the same robotic setup with minor adjustments to the placement of the UHF-RFID sensors. We observed the degree of divergence between the two rounds of robotic scans lies between $\pm 2 \mathrm{~dB}$ with a 0.89 correlation coefficient. Secondly, when the position of the UHF-RFID reader antenna changes, the pattern of the UHF-RFID sensor signals changes as well. Also, the direction in which the robot moves has no effect on reading the UHF-RFID sensor signals if the robot is equipped with a fixed UHF-RFID reader antenna setup. Further, the robotic speed has a limited effect on the UHF-RFID sensor signals. Lastly, when the UHF-RFID reader antenna is not nearby, each UHF-RFID sensor signal has a slightly unique signal pattern, even if the sensors are of identical type. We have seen the unique results from the two UHF-RFID antenna model in this work. Those data inputs will be used to improve the accuracy of in-pipe robot localization. 


\section{ACKNOWLEDGMENT}

This work was supported by the UTS FEIT Tech Lab Blue Sky Research Grant. Special thanks to Sydney Water funded project, "Development of sensor suites and robotic deployment strategies for condition assessment of concrete sewer walls" for making the robot available for this work.

\section{REFERENCES}

[1] K. Thiyagarajan, S. Kodagoda, R. Ranasinghe, D. Vitanage, and G. Iori, "Robust sensor suite combined with predictive analytics enabled anomaly detection model for smart monitoring of concrete sewer pipe surface moisture conditions," IEEE Sensors Journal, vol. 20, no. 15, pp. 8232-8243, Aug. 2020. [Online]. Available: https://doi.org/10.1109/jsen.2020.2982173

[2] N. Ulapane, K. Thiyagarajan, J. V. Miro, and S. Kodagoda, "Surface representation of pulsed eddy current sensor signals for improved ferromagnetic material thickness quantification," IEEE Sensors Journal, vol. 21, no. 4, pp. 5413-5422, Feb. 2021. [Online]. Available: https://doi.org/10.1109/jsen.2020.3034571

[3] K. Thiyagarajan, S. Kodagoda, R. Ranasinghe, D. Vitanage, and G. Iori, "Robust sensing suite for measuring temporal dynamics of surface temperature in sewers," Scientific Reports, vol. 8, no. 1, Oct. 2018. [Online]. Available: https://doi.org/10.1038/s41598-018-34121-3

[4] N. Giovanangeli, L. Piyathilaka, S. Kodagoda, K. Thiyagarajan, S. Barclay, and D. Vitanage, "Design and development of drillresistance sensor technology for accurately measuring microbiologically corroded concrete depths," in Proceedings of the 36th International Symposium on Automation and Robotics in Construction (ISARC). International Association for Automation and Robotics in Construction (IAARC), May 2019, pp. 735-742. [Online]. Available: https: //doi.org/10.22260/isarc2019/0099

[5] M. Ams, P. Ha, S. Taheri, S. Clark, M. J. Withford, H. Bustamante, J. Gonzalez, and L. Vorreiter, "Fibre optic temperature and humidity sensors for harsh wastewater environments," in 2017 Eleventh International Conference on Sensing Technology (ICST). IEEE, Dec. 2017, pp. 1-3. [Online]. Available: https://doi.org/10.1109/icsenst.2017. 8304493

[6] K. Thiyagarajan, S. Kodagoda, L. V. Nguyen, and R. Ranasinghe, "Sensor failure detection and faulty data accommodation approach for instrumented wastewater infrastructures," IEEE Access, vol. 6, pp. 56 562-56574, 2018. [Online]. Available: https://doi.org/10.1109/ access.2018.2872506

[7] K. Thiyagarajan, S. Kodagoda, N. Ulapane, and M. Prasad, "A temporal forecasting driven approach using facebook's prophet method for anomaly detection in sewer air temperature sensor system," in 2020 15th IEEE Conference on Industrial Electronics and Applications (ICIEA). IEEE, Nov. 2020, pp. 25-30. [Online]. Available: https://doi.org/10.1109/iciea48937.2020.9248142

[8] K. Thiyagarajan, P. Acharya, L. Piyathilaka, and S. Kodagoda "Numerical modeling of the effects of electrode spacing and multilayered concrete resistivity on the apparent resistivity measured using wenner method," in 2020 15th IEEE Conference on Industrial Electronics and Applications (ICIEA). IEEE, Nov. 2020, pp. 200-206. [Online]. Available: https://doi.org/10.1109/iciea48937.2020.9248217

[9] A. Gunatilake, L. Piyathilaka, A. Tran, V. K. Viswanathan, K. Thiyagarajan, and S. Kodagoda, "Stereo vision combined with laser profiling for mapping of pipeline internal defects," IEEE Sensors Journal, vol. 21, no. 10, pp. 11926-11934, 2021. [Online]. Available: https://doi.org/10.1109/jsen.2020.3040396

[10] A. Gunatilake, L. Piyathilaka, S. Kodagoda, S. Barclay, and D. Vitanage, "Real-time 3d profiling with RGB-d mapping in pipelines using stereo camera vision and structured IR laser ring," in 2019 14th IEEE Conference on Industrial Electronics and Applications (ICIEA). IEEE, Jun. 2019, pp. 916-921. [Online]. Available: https://doi.org/10.1109/iciea.2019.8834089

[11] E. Giannelos, E. Andrianakis, K. Skyvalakis, A. G. Dimitriou, and A. Bletsas, "Robust RFID localization in multipath with phase-based particle filtering and a mobile robot," IEEE Journal of Radio Frequency Identification, vol. 5, no. 3, pp. 302-310, Sep. 2021. [Online]. Available: https://doi.org/10.1109/jrfid.2021.3086759
[12] E. DiGiampaolo and F. Martinelli, "A robotic system for localization of passive UHF-RFID tagged objects on shelves," IEEE Sensors Journal, vol. 18 , no. 20, pp. 8558-8568, Oct. 2018. [Online]. Available: https://doi.org/10.1109/jsen.2018.2865339

[13] Z. Liu, Z. Fu, T. Li, I. H. White, R. V. Penty, and M. Crisp, "An ISAR-SAR based method for indoor localization using passive UHF RFID system with mobile robotic platform," IEEE Journal of Radio Frequency Identification, pp. 1-1, 2021. [Online]. Available: https://doi.org/10.1109/jrfid.2021.3097803

[14] A. Tzitzis, S. Megalou, S. Siachalou, T. G. Emmanouil, A. Kehagias, T. V. Yioultsis, and A. G. Dimitriou, "Localization of RFID tags by a moving robot, via phase unwrapping and non-linear optimization," IEEE Journal of Radio Frequency Identification, vol. 3, no. 4, pp. 216-226, Dec. 2019. [Online]. Available: https://doi.org/10.1109/jrfid.2019.2936969

[15] L. Catarinucci, S. Tedesco, and L. Tarricone, "Customized ultra high frequency radio frequency identification tags and reader antennas enabling reliable mobile robot navigation," IEEE Sensors Journal, vol. 13, no. 2, pp. 783-791, Feb. 2013. [Online]. Available: https://doi.org/10.1109/jsen.2012.2227715

[16] F. Bernardini, A. Buffi, D. Fontanelli, D. Macii, V. Magnago, M. Marracci, A. Motroni, P. Nepa, and B. Tellini, "Robotbased indoor positioning of UHF-RFID tags: The SAR method with multiple trajectories," IEEE Transactions on Instrumentation and Measurement, vol. 70, pp. 1-15, 2021. [Online]. Available: https://doi.org/10.1109/tim.2020.3033728

[17] A. Gunatilake, M. Galea, K. Thiyagarajan, S. Kodagoda, L. Piyathilaka, and P. Darji, "Using UHF-RFID Signals for Robot Localization Inside Pipelines," in 2021 16th IEEE Conference on Industrial Electronics and Applications (ICIEA). IEEE, Aug. 2021, pp. 1109-1114.

[18] B. Tao, H. Wu, Z. Gong, Z. Yin, and H. Ding, "An RFIDbased mobile robot localization method combining phase difference and readability," IEEE Transactions on Automation Science and Engineering, vol. 18, no. 3, pp. 1406-1416, Jul. 2021. [Online]. Available: https://doi.org/10.1109/tase.2020.3006724 